\title{
181. Sputtering of Metals by Disruptive Discharge in Magnetic Field.
}

\author{
By Hantaro Nagaoka and Tetsugoro Futagami. \\ Institute of Physical and Chemical Research.
}

(Rec. Dec. 6, 1927. Comm. by H. NAGAOKA, M.I.A., Dec. 12, 1927.)

The sputtering hitherto described ${ }^{1)}$ was made in air without the action of external electric or magnetic field; with some metals it takes place more violently when the electrodes are placed in strong magnetic field and discharged by the same process as before with a big condenser battery in the circuit.

For this purpose, the samples are brought in loose contact having an area of a few $\mathrm{mm}^{2}$, and heavy current is sent through them, instantaneously vaporizing the metals near the place of contact. A water-cooled electromagnet with pole-pieces of $80^{\circ}$ for giving nearly uniform field and end face of $1 \mathrm{~cm}$ diam, separated by an interferricum of $1 \mathrm{~cm}$, serves to give a field of about 30 kilogauss, the end faces of the poles being insulated by thick micanite plates. The contact face of the metals is in line with the axis of the poles. The spark gap for discharge is placed outside this space.

On passing the current, the contact surface of the sample is intensely heated, and luminous particles are shot off, but the greatest luminosity is confined to the neighbourhood of the interferricum. The sputtering thus produced in the magnetic field is not specially great with alkaline metals, but those of alkaline earths show remarkable features. Especially interesting are the tracks of particles sputtered from $\mathrm{Ti}, \mathrm{Cr}$, and $\mathrm{Mn}$; those from ferromagnetic metals are not however so conspicuous. The description of the particulars cannot here be given; only a rough sketch of different appearances of sputtering is attempted.

$\mathrm{Ti}, \mathrm{Cr}$, and $\mathrm{Mn}$ show sputtering of the type given in Fig. 1. The particles follow nearly straight course at the outset, but curve downwards after passing out of the field and approach the end of luminosity. Nearly all the particles show sudden change of curvature

1) Nagaoka, /Nukiyama a. Futagami : Proc. Imp. Acad. 3 (1927) 499. 
or are forked, showing that the particles are suddenly separated into several parts; the forks thus observed must be due to sudden change of momentum of the original particle. As the above metals are easily oxidized, the chemical action must be the principal cause of such abrupt changes in the tracks. There is close analogy with the tracks of recoil atoms in Wilson's experiment on radioactive changes. The appearance of luminous particles resembles that of sparks from the grinders or 'Senko-hanabi'); specially interesting are the tracks of long range particles in the present experiments as shown in Fig. 2 for $\mathrm{Cr}$.

The tracks of $\mathrm{Al}$ (Fig. 3) sputtered in magnetic field are characterised by showing e'rious trends towards the end. Instead of showing sharp bends and forks, they generally curl in a convoluted manner, and sometimes end quite abruptly. The luminescence, as indicated in the photographs, becomes intense near the tails, the intermediate course being sometimes obscure. Most probably the particles shot out do not oxidize at the moment of disruption, but take some time before they are acted by the oxygen of the air, and the brilliancy increases by the chemical change. The appearance of the long ranged particles can be gathered from Fig. 4. The resemblance with the tracks of $\beta$-particles in Wilson's experiment is evident at a glance. Among the curled tracks of $\mathrm{Al}$ particles are found beads which follow for a large number of times, apparently showing the successive fluctuations in luminosity. Such trails of light are also found in sputtered $\mathrm{Ca}$ particles and more conspicuously in $\mathrm{W}$ and Mo.

$\mathrm{W}$ and Mo are both remarkable for shooting out particles describing straight paths. The intensity generally decreases as they move outward, but sometimes sudden increase is noticed; this is apparently due to oxidation. In $\mathrm{W}, \mathrm{WO}_{2}$ is first formed and this is further oxidized to $\mathrm{WO}_{3}$; similar phenomenon can be observed with other elements. On magnifying the photographs, we found that the tracks of many long ranged particles are composed of dots, which sometimes number more than a hundred. This must be similar to the beaded tracks of $\mathrm{Al}, \mathrm{Ca}$ and $\mathrm{Mg}$, and calls for separate discussion.

With $\mathrm{Ce}$, the intensity changes are numerous, but the variation is not so intense as with other elements. The photographs show a number of protuberances in the course of the particles, which are generally followed by projections of small particles of luminous matter in the lateral direction, leading a straight course; this may be the

1) NAKAYA: Proc. Imp. Acad. 3 (1927) 510. 
effect of the separation of oxide coating formed on the surface of the projected particles.

The tracks of particles from other elements are various that space allotted to the communication does not permit us to give detailed description. The mode of projection of the particles is similar to electric explosion ${ }^{1)}$, but the paths are somewhat different, owing to the intense heating of the surface of contact and the action of strong magnetic force on the particles, most of which can be considered as heavy ions. Obviously the force acting on such ions is given by $e[H v]$, where $e$ is the charge, $v$ the velocity and $H$ the magnetic field; it acts in the direction normal to that of motion. Owing to the high temperature of the particles and the electric force at the place of contact, it is possible that the discharge results in the liberation of thermions, by which process the particles are likely to be positively charged. The force acting on the particles by the introduction of magnetic field is tolerably large, so that the remarkable effect of sputtering here described seems to be a natural consequence. It is however to be remarked that the space where the magnetic field acts should not be too large as the particles may describe nearly circular orbits and not issue out of the said space. Closer examination of the sputtering of alkaline metals will make this point clearer.

The sputtering seems to have some connection with the melting and boiling points, the closeness of which apparently increases the effect. The tracks are also influenced by the viscosity of the air and its sudden heating. The magnetic property of the metals plays a minor part, as the temperature of the sputtered particles are far above that at which the ferromagnetic quality is lost, as experiments with iron, cobalt and nickel seem to indicate.

1) Nagaoka, Futagami a. Machida: Proc. Imp. Acad. 2 (1926) 328. 


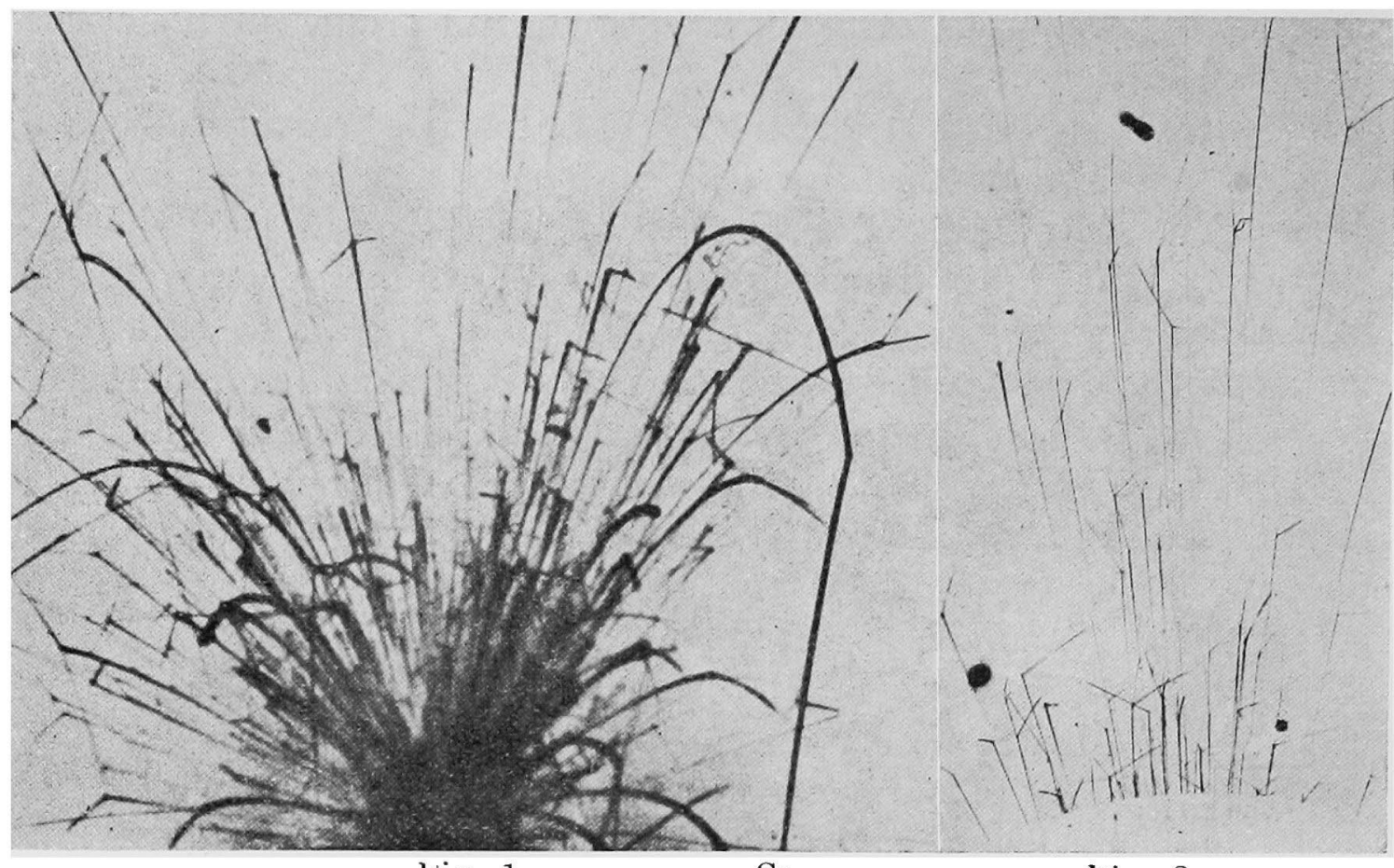

Fig. 1.

Fig. 2 .

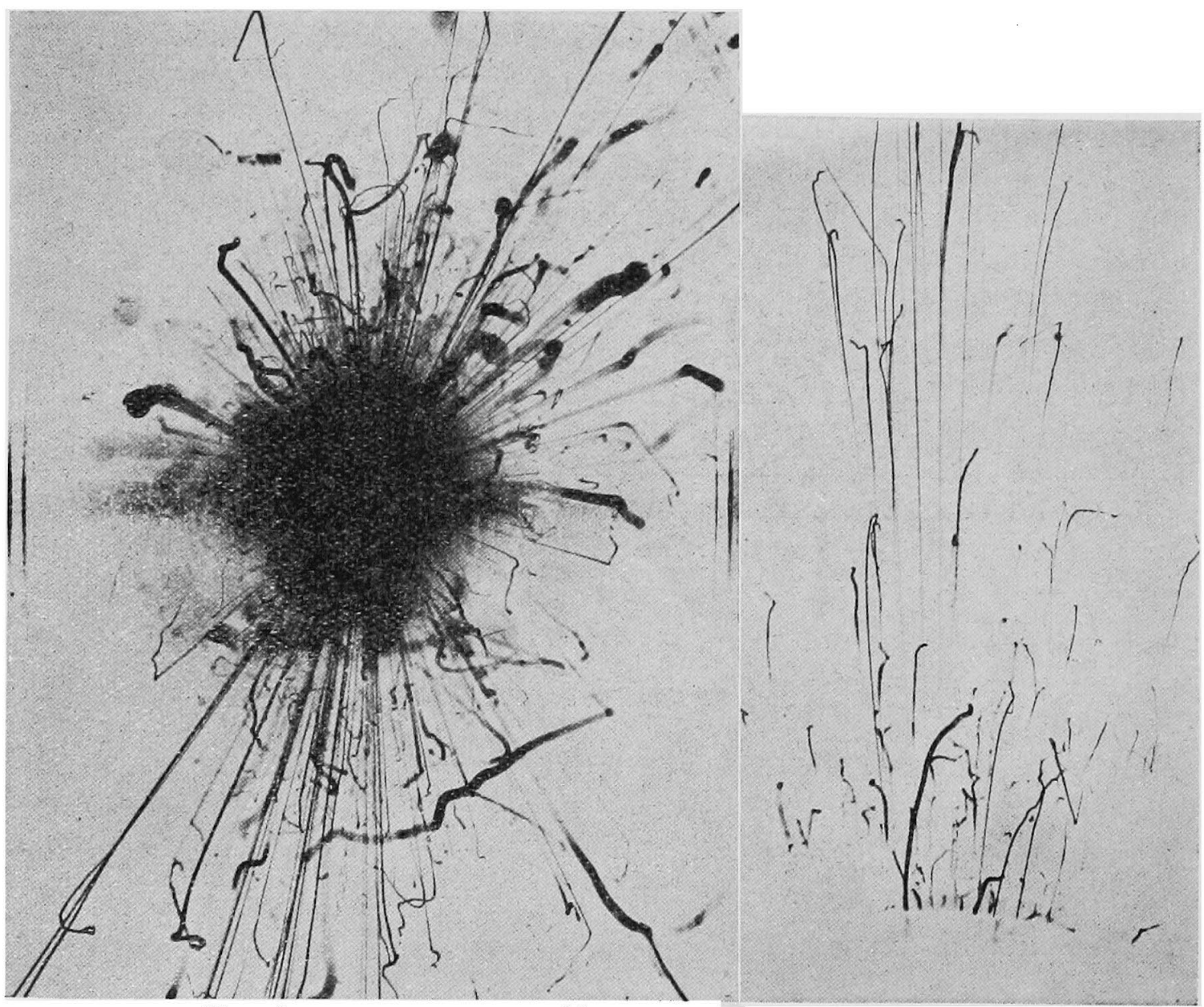

Fig. 4. 